Abb. 1 Mariendistel. Sie ist eine zentrale Heilpflanze in der Lebertherapie - nicht nur unterstützend bei chronisch-entzündlichen Lebererkrankungen, sondern auch als Kur bei Erkrankungen, die mit dem Leberstoffwechsel korrelieren. Quelle: Kirsten Oborny/Thieme Gruppe 


\section{Ein Auge auf die Nachbarschaft}

\section{Therapie von LEBER-GALLE-STÖRUNGEN unter Einbeziehung der benachbarten Organe Maria Lohmann}

\section{KURZ GEFASST}

1 Häufig sind Alkoholgenuss und Überernährung an Leber-GalleErkrankungen beteiligt, zunehmend jedoch auch Dysbiosen, Medikamente, Schadstoffe und Diabetes mellitus.

2 Neben der gängigen Leberdiagnostik liefert auch die Irisdiagnose wertvolle Hinweise, wobei gilt: Die Therapie sollte möglichst beginnen, bevor die Laborwerte auffällig werden.

3 Diätetik, Mariendistel, Artischocke, Probiotika, Selen und Zink zählen zum erfolgreichen Repertoire der naturheilkundlichen Leber-Galle-Therapie.

In der Praxis sprechen Leber-Galle-Erkrankungen meist gut auf naturheilkundliche Therapien an. Denn sie stärken das Regenerationsvermögen der Leber. Ernährungsumstellung und Phytotherapie bilden hierbei die wichtigsten Grundlagen. Bewährt haben sich zudem die Behandlung mit Homöopathie, Ausleitungs- und Umstimmungsverfahren sowie physikalische Maßnahmen wie der Heublumensack oder abendliche feucht-warme Leberwickel.

\section{Alle Leberstörungen belasten den Darm - und umgekehrt}

Leber und Galle stehen in so enger funktioneller und topografischer Beziehung zu Magen, Dünndarm und Pankreas, dass sich diese Organe wechselseitig stark beeinflussen. Daher wirken sich alle Störungen - aber auch Therapien - von Leber und Gallenblase auf Magen und Darm aus und umgekehrt, zum Beispiel beim Leaky-Gut-Syndrom.

In die Therapie sollte neben dem Darm bei entsprechenden Hinweisen auch die Bauchspeicheldrüse einbezogen werden. Ein besonderes Augenmerk gilt dabei Diabetikern. Denn ein erhöhter Blutzucker begünstigt die Neubildung von Fett in der Leber. Ein wichtiger Grundsatz nach HP Josef Karl lautet daher: Keine Diabetestherapie ohne Lebertherapie!

\section{Leberbelastung durch Dysbakterie: intakte Darmmikrobiota als Therapieziel}

Eine ungünstige Zusammensetzung der enteralen Bakterienzusammensetzung kann die Entstehung und den Verlauf chronischer Erkrankungen der Gallenblase, Gallenwege und Leber erheblich beeinflussen. Erfahrungsgemäß liegt häufig eine Dysbakterie vor, die toxische Gärungs- und Fäulnisprodukte freisetzt. Das belastet die Leber in ihrer Funktion als zentrales Entgiftungsorgan. In der überwiegenden Zahl der Fälle ist deshalb zusätzlich eine Darmsanierung beziehungsweise mikrobiologische Therapie nach einer Stuhluntersuchung erforderlich. Das gilt ebenso für die chronische Obstipation, bei der vermehrt belastende Substanzen wie Indol und Skatol entstehen. Diese gelangen über die Pfortader zur Leber und werden dort abgebaut. Auch bei Patienten mit Zöliakie sollten Leber und Galle in den Therapieplan einbezogen werden.

\section{Immer häufiger: nichtalkoholische Lebererkrankungen}

Die verbreitete Überernährung und Belastungen durch Medikamente (Antibiotika, Glukokortikoide), Hormone („Pille“), Schwermetalle, Schimmelpilzgifte und andere Toxine verursachen eine zunehmende Leberbelastung. Daher treten Störungen immer häufiger auch bei Patienten auf, die ansonsten keine typischen Risikofaktoren (Alkohol, hyperkalorische Ernährung) aufweisen. In vielen Fällen liegt eine latente Insuffizienz vor, die im Labor nicht nachweisbar ist.

$\mathrm{Zu}$ den häufigsten Lebererkrankungen in der Praxis zählen Fettleber, Hepatitis (Achtung: Behandlungsverbot infektiöser Hepatitis für Heilpraktiker nach IfSG!), Gallenbeschwerden wie 


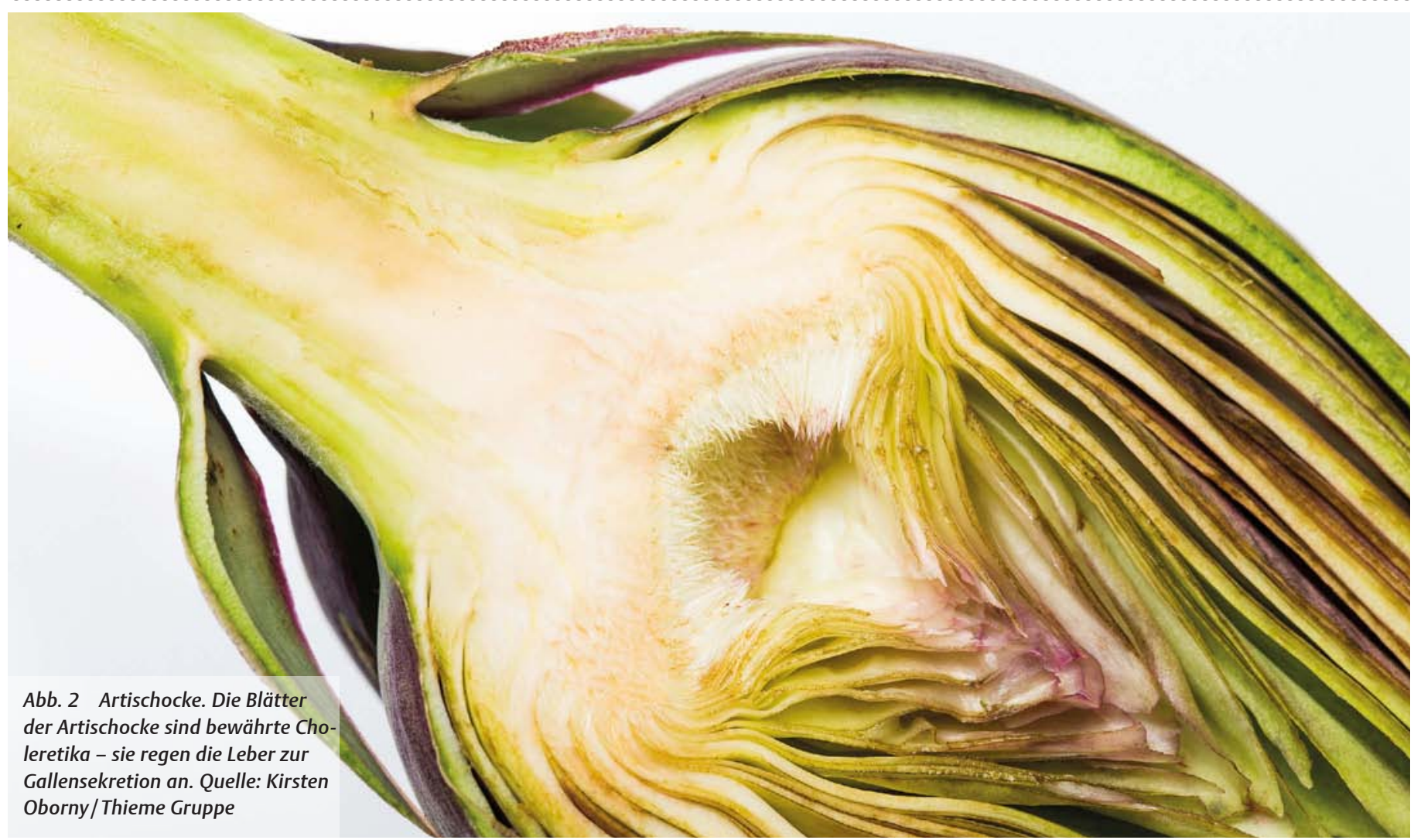

Cholelithiasis, Beschwerden nach Gallenblasenresektion sowie mit dem Leberstoffwechsel in Verbindung stehende Beschwerden wie Verdauungsstörungen, Kopfschmerzen oder Hautprobleme. Bei schweren Schäden wie Leberzirrhose kann die Naturheilkunde lediglich begleiten.

\section{Nicht auf pathologische Werte warten: Therapie frühzeitig beginnen}

Auch wenn die Leberwerte im Labor (noch) unauffällig sind, sollte bereits frühzeitig eine Lebertherapie eingeleitet werden. Denn gerade eine latente Insuffizienz ist im Labor meist nicht nachweisbar. Bei Frauen kann die jahrelange Einnahme der Pille die Leber belasten, da über sie der Abbau der Hormone erfolgt. Auch wenn diese oft jungen Patientinnen kaum Alkohol oder andere belastende Substanzen zu sich nehmen, kann eine Leberunterstützung sinnvoll sein.

\section{"Müdigkeit ist der Schmerz der Leber"}

Die belastete oder kranke Leber ruft zunächst keine Schmerzen hervor, da sich im Inneren des Organs keine Nerven befinden. Die Symptome einer Lebererkrankung treten deshalb oft erst spät auf, was eine frühzeitige Diagnose erschwert. Häufige unspezifische Symptome sind Müdigkeit („Müdigkeit ist der Schmerz der Leber“), Abgeschlagenheit, Schlafstörungen, Verdauungsbeschwerden und Blähungen. Klagt der Patient über ein Druck- oder Völlegefühl im rechten Oberbauch, könnte es sich um eine Spannung der Bindegewebskapsel aufgrund einer Lebervergrößerung handeln. Völlegefühl kann natürlich auch im Zusammenhang mit Erkrankungen der Gallenblase und Gallenwege stehen. Typisch sind weiterhin die Unverträglichkeit bestimmter - vor allem fetter Speisen, oft verbunden mit Übelkeit und Blähungen.

\section{Naturheilkundliche Diagnostik: Auf Zungenzeichen achten}

Bei Verdacht auf eine Leber- oder Gallestörung erfolgt die Basisdiagnostik in Form von Anamnese, körperlicher Untersuchung, Laboruntersuchungen mit den gängigen Leberenzymen und eventuell einer Stuhldiagnostik. Zusätzlich kann die Betrachtung der Zunge Aufschluss geben: Gelbliche Beläge in der hinteren Zungenhälfte weisen auf eine Leber-Galle-Störung, gelblich-bräunliche Beläge auf eine Darmstörung mit Leber-Galle-Beteiligung hin. Seitliche Zahneindrücke sind ebenfalls ein deutlicher Hinweis auf eine Leberbelastung (in der TCM wird der laterale Zungenrand der Leber und Gallenblase zugeordnet).

\section{TCM und Irisdiagnose: Was die Augen über die Leber erzählen}

Die TCM ordnet die Augen der Leber als Sinnesorgane zu. Eine nachlassende oder schwankende Sehschärfe wird im Zusammenhang mit Leberstörungen gesehen.

In der Irisdiagnose weisen die hämatogene (dyskratisch) und Mischkonstitution (biliär) auf eine Neigung zu Leber-Galle-Er- 
krankungen hin. Dies gilt auch für Schwächezeichen oder vaskularisierte Gefäße zwischen 7 und 8 Uhr in der rechten Iris. Aufhellungen in diesem Bereich treten bei akuten oder subakuten Entzündungen auf. Abdunkelungen kennzeichnen hingegen chronische Prozesse und Funktionseinschränkungen. Chronische Entzündungen der Gallenblase gehen oftmals mit einer Pigmentierung im Leber-Galle-Sektor einher.

Auch die Tageszeit, zu der Beschwerden möglicherweise regelmäßig auftreten, kann einen Hinweis auf funktionsgestörte Organe liefern. So deuten Schlafprobleme in der Zeit von 23 bis $1 \mathrm{Uhr}$ auf eine Störung der Galle, in der Zeit von 1 bis 3 Uhr auf eine Leberstörung hin.

\section{Fettleber als Diabetes- und Herz-Kreislauf-Risiko}

Eine Fettleber zeichnet sich durch eine zunehmende Ansammlung von Triglyzeriden in den Leberzellen aus. Dies kann unbehandelt zu Leberentzündungen führen. Häufig wird eine verfettete Leber von den betroffenen Patienten und selbst von Therapeuten nicht hinreichend ernst genommen.

Dabei kann eine nichtalkoholische Fettlebererkrankung (NAFLD = non-alcoholic fatty liver disease) eine Reihe von Fehlsteuerungen im Stoffwechsel verursachen und das Risiko für Dia-

\section{ÜBERBLICK}

\section{Wichtige Heilpflanzen für Leber und Galle}

Mariendistel: leberprotektiv, regenerierend, antihepatotoxisch

Artischocke: bei dyspeptischen Beschwerden, choleretisch, Absenken des LDL-Cholesterins, antioxidative Wirkung

Löwenzahn: cholagog, choleretisch, stoffwechselsteigernd

Wermut: Dyskinesien der Gallenwege, choleretisch, entkrampfend

Gelbwurz: choleretisch, cholekinetisch, entzündungshemmend

Schafgarbe: choleretisch, spasmolytisch, antibakteriell

Pfefferminze: cholagog, choleretisch, spasmolytisch

Wegwarte: bei Dyspepsie, cholagog, stoffwechselregulierend

\section{Hinweise zur Einnahme}

- Bitterstoffe 20-30 min vor dem Essen einnehmen, zum Beispiel Amara Tropfen Weleda 10 Tr.

- Bei Leber- und Gallemitteln aufgrund des Biorhythmus der Organe eine Dosierung zur Nacht geben.

- Bei der Anwendung von anregenden Gallemitteln sind Gegenanzeigen wie Verschluss der Gallenwege zu beachten.

- Bei allen Lebererkrankungen ist Alkohol komplett zu meiden (Alkoholgehalt von Arzneimitteln prüfen). 
betes mellitus und Herz-Kreislauf-Erkrankungen deutlich steigern. Bei Nichtbehandlung kann sie in die entzündliche nichtalkoholische Steatohepatitis (NASH = non-alcoholic steatohepatitis) übergehen, die letztlich zur Leberzirrhose führt. Häufig ist die nichtalkoholische Fettleber auf Fehlernährung zurückzuführen. Deshalb findet man sie vornehmlich bei Übergewichtigen. Oft wird jedoch übersehen, dass auch Schlanke betroffen sein können, vor allem Diabetiker.

Merke: Auch schlanke Menschen können eine Fettleber entwickeln. Dies betrifft vor allem Diabetiker.

\section{Phytotherapie: Anregen, schützen, entgiften}

Heilpflanzen zur Behandlung von Leber-Galle-Störungen nehmen eine zentrale Rolle im Therapieplan ein. Vorwiegend handelt es sich um Bitterstoffdrogen, die neben einer Tonisierung von Leber und Galle die gesamte Verdauungstätigkeit positiv beeinflussen. Cholagoga regen die Gallensaftproduktion an, Choleretika fördern den Gallenfluss.

Allgemein ist eine deutliche Unterscheidung zwischen Leberund Gallenmitteln nur schwer möglich, denn die Pflanzen besitzen in der Regel einen Einfluss auf beide Organe. Sie werden üblicherweise bei funktionellen und organischen Veränderungen der Gallenwege (vor allem Gallenwegsdyskinesie), Gallengrieß, funktioneller Oberbauchschwäche, Obstipation und zum Beispiel Fettleber eingesetzt.

Artischocke steigert den Gallenfluss

Zu den wichtigsten Choleretika zählen die Blätter der Artischocke mit ihrer entkrampfenden Wirkung, zum Beispiel Schoenenberger Naturreiner Heilpflanzensaft Artischocke $3 \times$ tgl. $10 \mathrm{ml}$ oder Hepar-SL ${ }^{\circledR} 320 \mathrm{mg}$ (Fa. MCM Klosterfrau) $2 \times$ tgl. 2 Kps. Dies gilt auch, wenn Patienten über Druckgefühl im rechten Oberbauch, Völlegefühl oder Blähungen klagen oder möglicherweise ein erhöhter Cholesterinwert vorliegt. Artischockenpräparate werden zudem eingesetzt, um Gallensteinrezidiven vorzubeugen.

\section{Zentrale Leberpflanze: Mariendistel zur Zellregeneration}

Eine einzigartige leberspezifische Wirkung besitzen die hepatoprotektiven Mariendistelfrüchte. Diese werden üblicherweise als hochdosiertes Fertigpräparat verordnet, zum Beispiel Silymarcur ${ }^{\circledR}$ (Fa. Rodisma-Med) $3 \times$ tgl. 1 Tbl., Legalon ${ }^{\circledR}$ forte (Fa. Meda) $3 \times$ tgl. 1 Kps. oder Silymarin-Loges ${ }^{\circledR} 3 \times$ tgl. 1 Tbl., jeweils als kurmäßige Einnahme über mehrere Wochen bis Monate. Silymarin ist ein Flavonoidgemisch mit antioxidativen und zellprotektiven Eigenschaften. Die Mariendistel ist sehr gut verträglich, erhöht signifikant die Belastbarkeit der Leberzellen und fördert ihre Regenerationskraft. Damit ist sie eine zentrale Heilpflanze in der Lebertherapie und wirkt unterstützend bei chronisch-entzündlichen Lebererkrankungen, aber auch als Kur bei Erkrankungen, die mit dem Leberstoffwechsel korrelieren. Dazu zählen unter anderem Erschöpfungssyndrome, depressive Verstimmungen und dermatologische Erkrankungen, insbesondere wenn sie mit Juckreiz einhergehen.
Zu den pflanzlichen Lebertherapeutika zählen weiterhin die aus Sojabohnen gewonnenen Sojaphospholipide mit ihren ungesättigten Fettsäuren. Sie besitzen eine cholesterinsenkende Wirkung und unterstützen die Leistungsfähigkeit der Leber. Sie sind insbesondere für Leberpatienten mit Hypercholesterinämie angezeigt und haben sich auch als Leberschutz nach Antibiotikaeinnahme oder zur Vorbeugung von Gallensteinen bewährt, zum Beispiel Lipidavit ${ }^{\circledR}$ SL (Fa. Rodisma-Med) $3 \times$ tgl. 2 Kps.

\section{Synergie nutzen: Bewährte pflanzliche Kombinationen}

$\mathrm{Zu}$ empfehlen sind auch Kombinationen wie Mariendistel mit Gelbwurz, zum Beispiel Bilisan ${ }^{\circledR}$ Duo (Fa. Repha) $3 \times$ tgl. 1 Tbl. oder Mariendistel mit Artischocke wie Salus Alepa ${ }^{\circledR}$ Mariendistel BioLeber-Tonikum $1 \times$ tgl. 10-15 ml.

Als eine weitere bewährte pflanzliche Kombination gelten Erdbeerblätter und Weinlaub, zum Beispiel in Hepatodoron ${ }^{\circledR}$ (Fa. Wele-

\section{FALLBEISPIEL}

\section{Aus der Patientenakte}

Eine 39-jährige Patientin, Briefzustellerin, leidet unter diffusen Oberbauchbeschwerden.

Symptome: Völlegefühl nach den Mahlzeiten, Druckschmerz im rechten Oberbauch, Aufstoßen, Fettunverträglichkeit, zeitweise Obstipation. Laborparameter und Sonografie der Hausärztin blieben ohne pathologischen Befund.

Diagnostik: Der gelblich-braune Zungenbelag mit seitlichen Zahneindrücken deutet auf eine Darmbelastung mit Leber-Galle-Beteiligung hin. Auf der Iris ist bei seitlichem Lichteinfall ein feiner Ölfilm zu erkennen, was auf eine Dysbakterie hinweist. Das Hautkolorit ist geringfügig ins Gelbliche verschoben. Die Patientin isst gerne Süßigkeiten.

\section{Therapie:}

- Milde Ableitungsdiät über drei Wochen, anschließend überwiegend laktovegetabile Ernährung

- Regulierung der Darmmikrobiota mit Kanne Brottrunk $2 \times$ tgl. $100 \mathrm{ml}$ über 4 Wochen

- Zur Anregung des Gallenflüssigkeit Choleodoron ${ }^{\circledR} 3 \times$ tgl. 10 Tr.

- Zur Leberregeneration Silymarin-Loges ${ }^{\circledR} 3 \times$ tgl. 1 Drg. über 6 Wochen

- Heublumensack (Apotheke) auf den rechten Oberbauch

Weiterer Verlauf: Nach zehn Tagen waren die Beschwerden verschwunden. Die milde Ableitungsdiät wurde von der Patientin sehr gut vertragen. Die sich anschließende laktovegetabile Kost sowie die Wiederherstellung der physiologischen Darmmikrobiota verstärkten das subjektive Wohlbefinden der Patientin. Durch die Ernährungsumstellung und die phytotherapeutischen Mittel verschwand auch die gelegentlich auftretende Obstipation. Die Patientin ist bis heute beschwerdefrei. 
da): $3 \times$ tgl. 2 Tbl. vor den Mahlzeiten langsam zerkauen sowie abends vor dem Schlafengehen. Das Mittel ist hilfreich zur Anregung der Lebertätigkeit, bei Obstipation sowie bei Ekzemen, die mit dem Leberstoffwechsel in Verbindung stehen. Die Bildung der Gallenflüssigkeit stimulieren unter anderem Artischocke und Kurkuma, zum Beispiel Choleodoron ${ }^{\circledR}$ Tr. (Fa. Weleda) 2- bis 4-mal tgl. 5-15 Tr.

\section{Wertvolle Ergänzung: Homöopathische Komplexmittel}

Bewährte homöopathische Komplexmittel sind zum Beispiel Hepeel ${ }^{\circledR} \mathrm{N}$ Tbl. (Fa. Heel) $3 \mathrm{x}$ tgl. 1 Tbl. (im Mund zergehen lassen), Aranicyn ${ }^{\circledR}$ Leber-Gallemittel (Fa. Weber \& Weber) 3 x tgl. 5 Tr. oder Ceres Taraxacum comp. Leber-Galle Tropfen 3 x tgl. 5 Tr.

Merke: Das größte Fettleberrisiko geht von Alkohol, Medikamenten, Übergewicht, Überernährung und rasch resorbierbaren Zuckern aus.

\section{Mit Ernährung und Diätetik den Verlauf umkehren}

Eine Fettleber ist bei Ausschalten der auslösenden Noxen (Alkohol, Medikamente, Übergewicht etc.), angepasster Ernährung und naturheilkundlichen Maßnahmen selbst noch im fortgeschrittenen Stadium teilweise oder vollständig reversibel. Der Patient sollte allerdings vom Alkohol nicht auf Fruchtsäfte, Limonaden oder Süßigkeiten umsteigen, da die darin enthaltenen rasch resorbierbaren Zucker ebenfalls die Leberverfettung begünstigen.

Merke: Alle rasch resorbierbaren Kohlenhydrate, insbesondere Glukose, Fruktose und Saccharose, belasten die Leber und sollten eingeschränkt werden.

Weißmehlprodukte sollten durch Vollkornvarianten ersetzt werden und Mahlzeiten generell nicht zu üppig ausfallen, um die Leber nicht innerhalb kurzer Zeit mit einer großen Fettmenge zu überlasten. Günstig ist eine niedrig-glykämische Ernährung mit 
viel Gemüse, Salaten, hochwertigen Pflanzenölen und mehrfach ungesättigten Fettsäuren.

\section{Gewichts- und Fettreduktion: Nicht zu viel des Guten}

Überernährung, insbesondere mit Kohlenhydraten wie Fruktose und Stärke, steht neben Alkohol an der Spitze der Ursachen einer Fettleber. Man spricht daher von Mastfettleber. Eine Gewichtsabnahme sollte jedoch langsam erfolgen, also nicht mehr als $500 \mathrm{~g}$ pro Woche betragen. Höhere Werte sind bei einer Fettleber kontraindiziert. Denn beim Abbau größerer Körperfettmengen kommt es zu einem vermehrten Zustrom von Fettsäuren und damit zu einem noch höheren Fettgehalt der Leber.

Bei Diäten mit starker Fettbeschränkung greifen die Betroffenen in der Regel vermehrt auf Kohlenhydrate zurück. Das belastet wiederum die Leber. Als schonend gilt hingegen die milde Ableitungsdiät, eine sanfte Form der F.-X.-Mayr-Diät. Wirksam ist auch eine zweitägige Haferkur (Haferbrei, mit Wasser aufgekocht). Strenge Fastenkuren wie Heilfasten gelten dagegen als Risikofaktor für Cholelithiasis.

\section{Mikronährstoffe: Meist Mangel an B-Vitaminen, Zink und Selen}

Lebererkrankungen erfordern ganz besonders eine ausreichende Versorgung des Körpers mit Vitaminen und Mineralstoffen. Neben dem Mangel an Vitaminen - insbesondere B-Vitaminen - zeigen Untersuchungen an Patienten mit Lebererkrankungen gehäuft einen Mangel an Zink und Selen. Als Substitution bieten sich hier

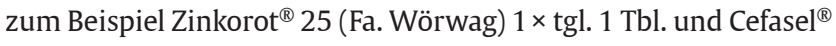
100 (Fa. Cefak) $1 \times$ tgl. 1 Tbl. an.

Die Darmmikrobiota spielt für das Auftreten und den Verlauf verschiedener Lebererkrankungen eine wichtige Rolle (siehe S. 28), die immer besser erforscht wird. Probiotika unterstützen das Mikrobiom und damit auch die Leber, zum Beispiel SymbioLact $^{\circledR}$ (Fa. Symbiopharm), Innovall ${ }^{\circledR}$ AID (Fa. Weber \& Weber) oder MyBiotik ${ }^{\circledR}$ Protect (Fa. nutrimmun), jeweils $1 \times$ tgl. 1 Portionsbeutel. Insbesondere natürlich vorkommende Milchsäurebakterien wie Bifidobakterien und Laktobazillen unterstützen den Aufbau einer gesunden Darmmikrobiota, entlasten die Leber und fördern die Ammoniakausscheidung über den Darm. Sie finden sich auch in gesäuerten Lebensmitteln wie Kefir, Frischkost-Sauerkraut, fermentiertem Gemüse oder Brottrunk.

\section{Leberentlastung durch Entgiftung im Darm}

Ein spezieller Kalium-Eisen-Phosphat-Citrat-Komplex, zum Beispiel Gelum ${ }^{\circledR}$-Tropfen (Fa. Dreluso) alkoholfrei $3 \times \operatorname{tgl} .2 \mathrm{ml}$ mit Wasser, bindet im Verlauf der Darmpassage das Verdauungsgift Ammoniak und entlastet somit die Leber. Anwendungsgebiete sind laut Hersteller Leberstörungen einschließlich schwerer Formen wie Leberzirrhose mit minimaler hepatischer Enzephalopathie.
Zur Entgiftung und Entlastung bieten sich auch Kombinationen aus Diosmektit, Myrrhe und Weihrauch an, zum Beispiel Symbio ${ }^{\circledR}$ detox Pulver (Fa. Symbiopharm) $1 \times$ tgl. 1 Stick. Sie können toxische Stoffe wie Schwermetalle und Bakterientoxine binden, die Darmschleimhaut beruhigen (auch bei Diarrhö) und die Leber entlasten.

\section{Mit der Leberfunktion verbessern sich diverse Störungen}

Die Naturheilkunde berücksichtigt die zentrale Aufgabe der Leber, indem sie leberkräftigende Maßnahmen sehr häufig im Behandlungsplan ihrer Patienten vorsieht. Dies gilt auch bei Erkrankungen, deren Ursache und Entstehung scheinbar außerhalb der Verdauungsorgane liegen, zum Beispiel Allergien, Hauterkrankungen (insbesondere Ekzeme), Rheuma sowie Kopfschmerzen und Migräne, aber auch Neigung zu depressiven Verstimmungen und Venenprobleme. Erfahrungsgemäß bessern sich viele dieser Erkrankungen, wenn die Entgiftungsfunktion der Leber gestärkt wird. Beispielsweise kann bei hepatogen bedingten Kopfschmerzen oder Migräne zusätzlich die frühzeitige Einnahme von Taraxacum, Chelidonium und Mariendistel (zum Beispiel Hepar Hevert ${ }^{\circledR}$ Lebertabletten, bis zu $6 \times$ tgl. alle 30 min 1 Tbl.) den Anfall verhindern oder abmildern (alternativ ein ähnliches Präparat als Injektion geben). Im Rahmen einer Entgiftungstherapie werden Lebermittel auch vielfach mit Nieren- und Lymphmitteln kombiniert.॰

Dieser Artikel ist online zu finden:

http://dx.doi.org/10.1055/a-1193-6252

\section{目 Literatur}

[1] Bierbach E, Hrsg. Naturheilpraxis heute. Lehrbuch und Atlas. 6. Aufl. München: Urban \& Fischer bei Elsevier; 2019

[2] Karl J. Neue Therapiekonzepte für die Praxis der Naturheilkunde. München: Pflaum; 1995

[3] Lohmann M. Einstieg in die Naturheilpraxis. 3. Aufl. München: Urban \& Fischer; 2007

[4] Lohmann M. Der Basen-Doktor. 3. Aufl. Stuttgart: Trias; 2017

[5] Lohmann M. Laborwerte verstehen. 6 Aufl. Murnau: Mankau; 2020

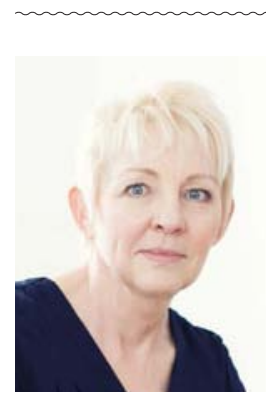

\section{HP MARIA LOHMANN}

Maria Lohmann ist Heilpraktikerin mit den Schwerpunkten Ernährung und Säure-Basen-Haushalt, Phytotherapie und klassische Naturheilverfahren. Sie ist Autorin zahlreicher medizinischer Bücher und Ratgeber zu verschiedenen Gesundheitsthemen und auBerdem Mitautorin des Standardwerks „Naturheilpraxis heute“.

Internet: www.maria-lohmann.de/ E-Mail: Maria.Lohmann@t-online.de 